\title{
Studies on the Magnetic Center of the Mu2e Solenoid System
}

\author{
M. L. Lopes, G. Ambrosio, M. Buehler, R. Coleman, D. Evbota, \\ V. Khalatian, M. Lamm, G. Moretti, T. Page, M. Tartaglia, Fermilab, \\ J. Miller, Boston University
}

\begin{abstract}
The definition of the magnetic center in the Mu2e solenoid system is not trivial given the $S$-shaped nature of the transport solenoid (TS). Moreover, due to the fringe field of the larger bore adjacent magnets - production solenoid (PS) and the detector Solenoid (DS) - the magnetic center does not coincide with the geometric center of the system. The reference magnetic center can be obtained by tracking a low momentum charged particle through the whole system. This paper will discuss this method and will evaluate the deviations from the nominal magnetic center given the tolerances in the manufacturing and the alignment of the coils. Methods for the correction of the magnetic center will also be presented.
\end{abstract}

Index Terms-Solenoid, Superconducting Magnets, Beam Transport

\section{INTRODUCTION}

$\mathrm{T}$ $\mathrm{HE} \mathrm{Mu} 2 \mathrm{e}$ experiment [1] proposes to measure the ratio of the rate of the neutrino-less, coherent conversion of muons into electrons in the field of a nucleus, relative to the rate of ordinary muon capture on the nucleus. The conversion process is an example of charged lepton flavor violation, a process that has never been observed experimentally. The conversion of a muon to an electron in the field of a nucleus occurs coherently, resulting in a monoenergetic electron (105 MeV) near the muon rest energy that recoils off of the nucleus in a two-body interaction. At the proposed Mu2e sensitivity there are a number of processes that can mimic a muon-to-electron conversion signal. Controlling these potential backgrounds drives the overall design of Mu2e. The overview of the Mu2e experiment can be seen in Fig 1. It is primarily formed by three large solenoid systems: the production solenoid (PS) [2] the transport solenoid (TS) [3] and the detector solenoid (DS) [4]. The muon beam is created by an $8 \mathrm{GeV}$, pulsed beam of protons striking a production target.

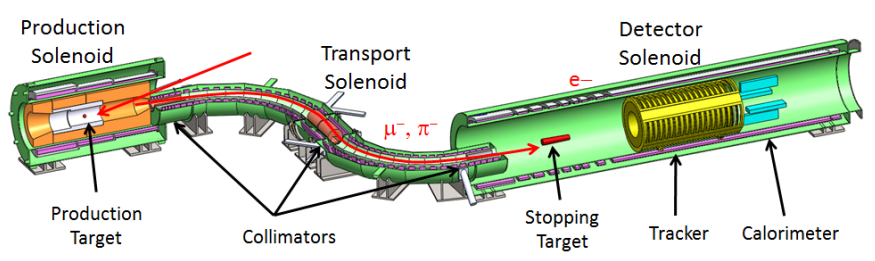

Fig. 1. The Mu2e experiment overview

Manuscript received July 16, 2013. Work supported in part by FRA under DOE Contract DE-AC02-07CH11359.

M. L. Lopes - Fermi National Accelerator Laboratory, Batavia, IL 60510 USA (mllopes@fnal.gov).
The negative magnetic field gradient in the PS creates a mirror effect on the charged particles produced after the collision, pushing the beam downstream. This beam is then transported by the TS. The three collimators in TS provide charge and momentum selection. In the DS, the muon beam is stopped in the stopping target and the $105 \mathrm{MeV}$ electrons from the conversion are detected in the tracker.

The magnetic system is formed by 3 coils for PS, 52 Coils for TS and 11 coils for DS. Each subsystem is in a separate cryostat module. TS is divided into two cryostats (named TSu and TSd).

\section{MAGNETIC CENTER VERSUS GEOMETRIC CENTER}

In general, straight solenoids have a well-defined magnetic axis. The magnetic axis is important because it determines the beam center. Very often, because of limitation in the construction, the magnetic axis does not coincide with the geometric center of a straight solenoid. In general, magnetic measurements should be carried out to relate them.

The magnetic center of the Mu2e solenoid system cannot be easily determined, given the S-shape nature of the TS. Fig. 2 shows, schematically, the difference between the geometric center of the coils and the magnetic center. Just like in a straight solenoid, the beam follows the magnetic center of the TS. The knowledge of that is crucial because the experiment relies on collimators to make both charge and momentum selection. If the beam is off-set with respect to a collimator, that may impact the muon transmission through the channel.

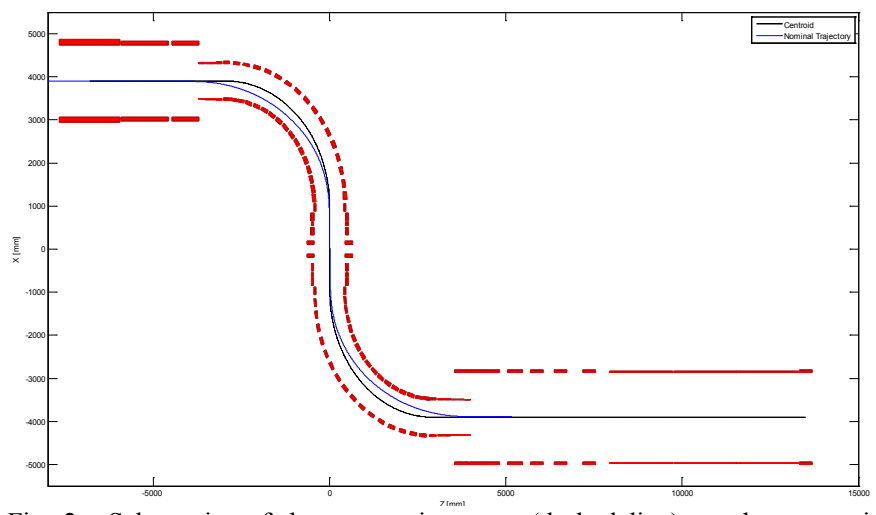

Fig. 2. Schematics of the geometric center (dashed line) vs. the magnetic center (solid line). The differences between the two were exaggerated for clarity. 


\section{LOW-MOMENTUM PARTICLE TECHNIQUE}

The magnetic center can be determined by tracking a lowmomentum charged particle (LMCP). The vertical drift is relatively small; therefore it stays close to the geometric center. Fig 3 shows the track of three different momentum particles through the magnetic system with respect to its geometric center.
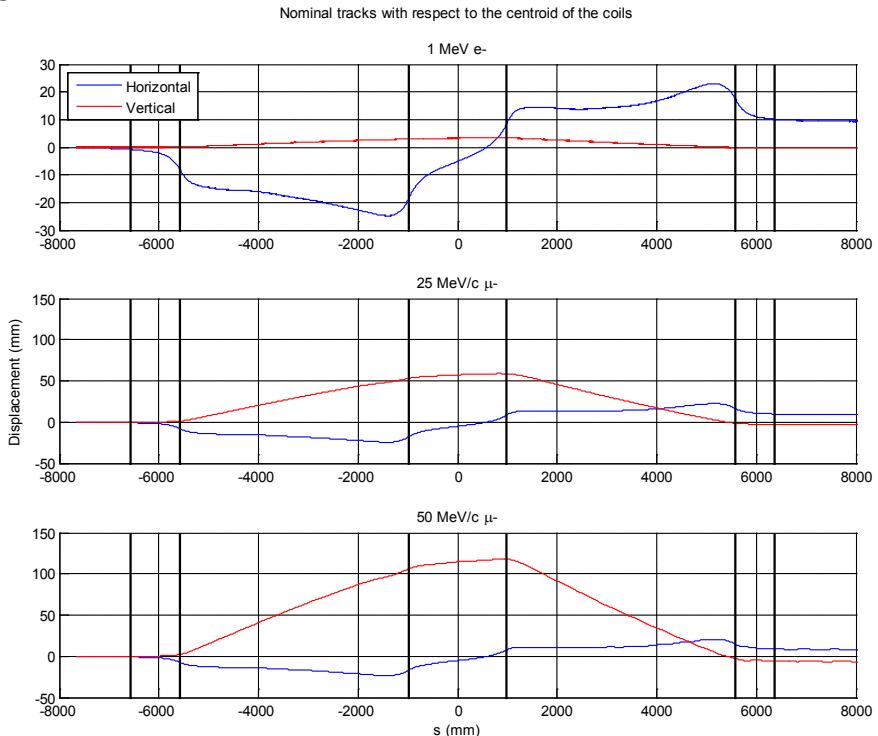

Fig. 3. Track of particles through the TS. Top: $1 \mathrm{MeV}$ e, Middle: $25 \mathrm{MeV} / \mathrm{c}$ $\mu^{-}$, Bottom: $50 \mathrm{MeV} / \mathrm{c} \mu$.

Figure 4 shows the transverse cross section of the muon beam (simulated) at the entrance of the third collimator (located at the last TS straight section). In red is the result before corrections in the toroidal magnetic system. As can be seen, the center of the beam is displaced by $100 \mathrm{~mm}$. Taking into consideration that the collimator radius is $150 \mathrm{~mm}$, a significant portion of the beam will be lost. This problem is caused by the fringe field of the adjacent solenoids (PS and DS) extending into the curves. This can be corrected by applying an extra yaw angle $(\sim 1$ degree $)$ on the coils that compose the curves (Fig 5). In blue is the result after the magnetic design was corrected.

This simulation, using G4Beamline [5], takes a relatively long time $(\sim 1 \mathrm{~h})$. The tracking of a single $\mathrm{MeV} \mathrm{e}^{-}$is very fast $(<1 \mathrm{~min})$ and was sufficient to correct the beam position.
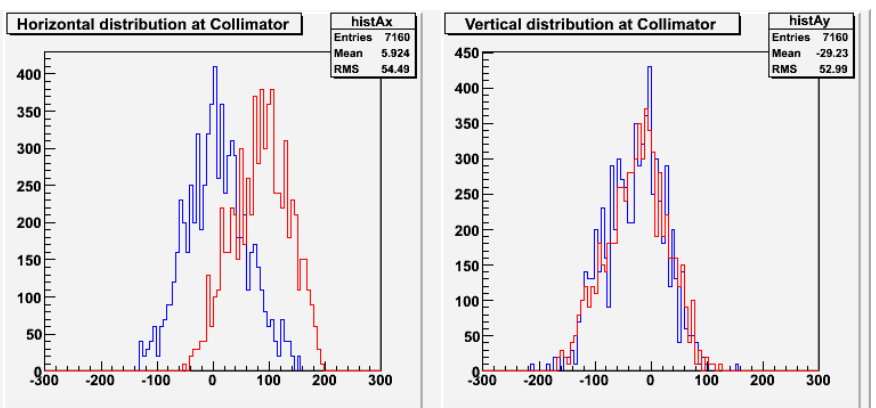

Fig. 4. The transverse cross section of the muon beam (simulated) at the entrance of the third collimator. Red: before the correction; blue: after the correction.
This technique can be used to study the geometrical tolerances of the magnetic system. Simulations of three different energy particles going through the transport solenoid were done. In each simulation a different random error was applied to each coil. The difference between the track in the perturbed state and at the nominal position is computed. The process is repeated 100 times for statistics. Average and standard deviations are calculated at each point of the track.

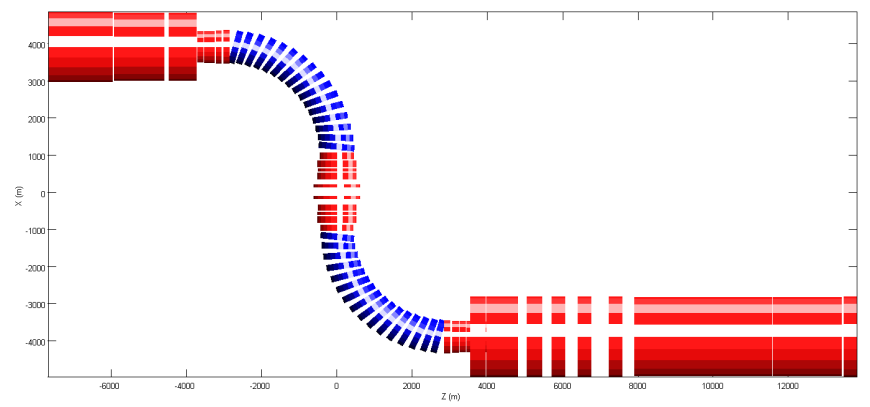

Fig. 5. Mu2e solenoid system. The coils in blue represent the ones with extra yaw of $\sim 1^{\circ}$ (the picture shows an exaggerated effect for clarity).

Figures 6 shows the standard deviation as function of the positions, when a random error of up to $5 \mathrm{mrad}$ is applied to the pitch angle of each coil. The pitch angle only affects the vertical position of the magnetic center. Likewise, yaw errors only affect the horizontal displacement.

As can be seen, the relative change of the beam position is independent of the particle momentum. Therefore, the standard deviation of a LMCP can be used as an indication of the displacement of the magnetic center. The advantage is that LMCPs have a very small pitch compared to the general dimensions of the magnets and the strength of the TS magnetic fields (from 2.5 to $2 \mathrm{~T}$ ). Hence the errors associated with the calculation of the magnetic center with respect to the geometric center are minimized.
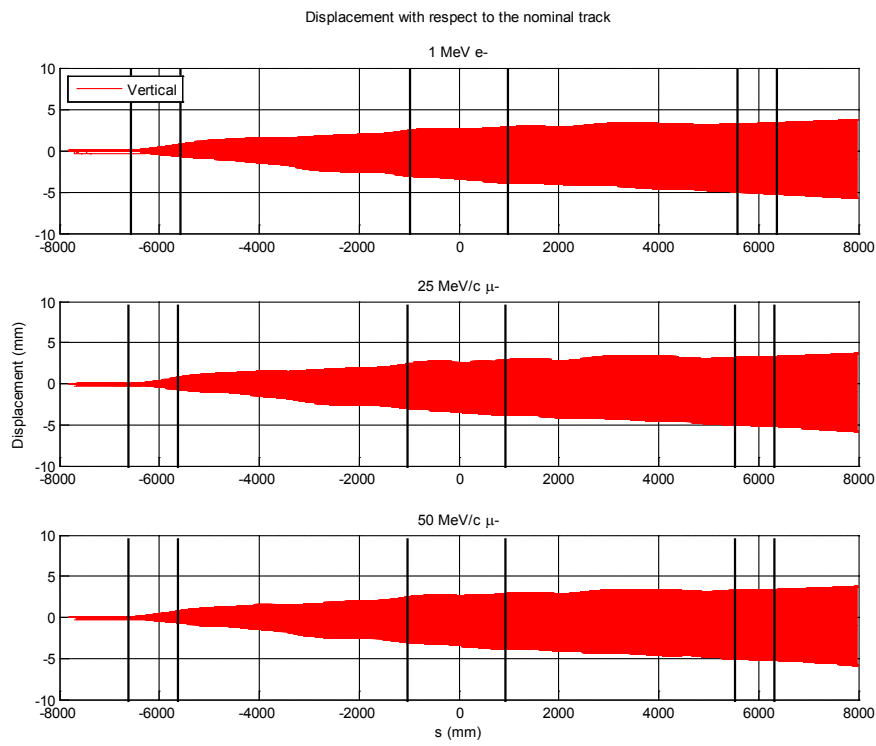

Fig. 6. Standard deviation as function of the trajectory of the perturbed coils system when random errors of up to $5 \mathrm{mrad}$ are present.

This technique was applied to study the sensitivity of three translational errors of the coils $(\mathrm{X}, \mathrm{Y}$ and $\mathrm{Z})$ and the two 
rotation angles (yaw and pitch). The errors associated with the rotations are more critical for the displacement of the magnetic center. The mechanical specifications for the coils placement were limited to be smaller than $0.2^{\circ}$. In that case the maximum RMS deviation is limited to $\sim 3 \mathrm{~mm}$.

\section{VERTICAL ALIGNMENT}

As mentioned before, one of the functions of TS is momentum and charge selection. The first curve in TS will drift the beam vertically; the drift is proportional to momentum of the particles. Given the orientation of the field, negative particles drift upward and positive particles drift downward. After the first curve, the beam goes through an asymmetric collimator (Fig 7). The second curve of TS brings the beam back to the center.

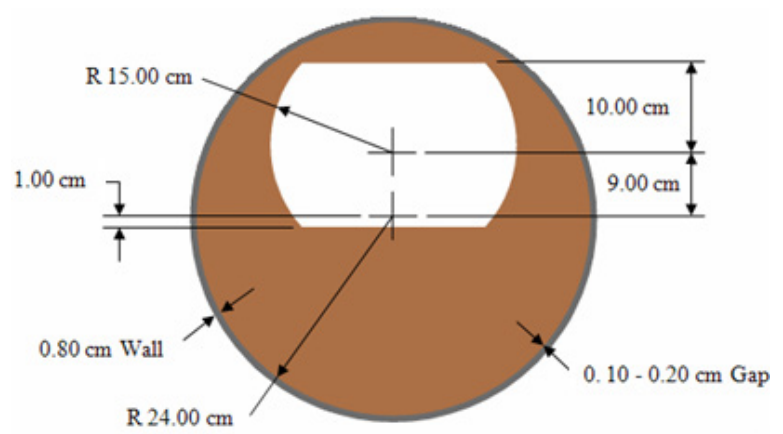

Fig. 7. Asymmetric collimator located in between the two curves.

The vertical alignment of the magnetic center is especially critical in the middle collimator region. Normally, $105 \mathrm{MeV}$ electrons coming from the production target are intercepted by the middle collimator. Studies have shown that misalignments may allow these electrons to go through the collimator and make their way to the detector, mimicking the signal from the conversion. In that case, the beam needs to be displaced upwards so that the $105 \mathrm{MeV}$ electrons hit the upper region of the collimator.

\section{A. Gravity supports}

In the previous section the track of a LMCP was used to determine the mechanical tolerances due to random errors on the positioning of the coils. The same technique can be used to study the mechanical tolerances that must be achieved by each TS cryostat.

Figure 8 shows the TSu cold mass with its radial, axial and gravity supports. The present design has three pairs of gravity supports (TS1, TS2 and TS3). The gravity supports can be used to intentionally misalign the coils, that is, introduce a systematic pitch angle error to affect the vertical displacement of the magnetic center on the middle collimator. Table I summarizes the results of the shift in vertical magnetic center as function of different gravity supports misalignments.
TABLE I

Vertical Magnetic CENTER DisPlaCEMENT AS FUNCTION OF THE GRAVITY SUPPORTS MISALIGNMENT. UNITS ARE mm.

\begin{tabular}{ccccc} 
TS3 & 0 & 10 & 20 & 30 \\
\cline { 2 - 5 } & & & & \\
\hline-4.0 & 6.4 & 12.7 & 19.0 \\
-8.0 & 2.3 & 8.7 & 15.0 \\
-11.9 & -1.6 & 4.7 & 11.0 \\
& -5.6 & 0.7 & 7.0 \\
\hline
\end{tabular}

The maximum displacement of $19 \mathrm{~mm}$ is achieved when the TS3 gravity support is lifted (or lowered) by $30 \mathrm{~mm}$ and the TS1 is left at the nominal elevation. The disadvantage of this method is that solenoids naturally align their axis. Therefore a centering force of $-68 \mathrm{kN}$ will appear. This amount can be handled by the gravity supports (in either direction).

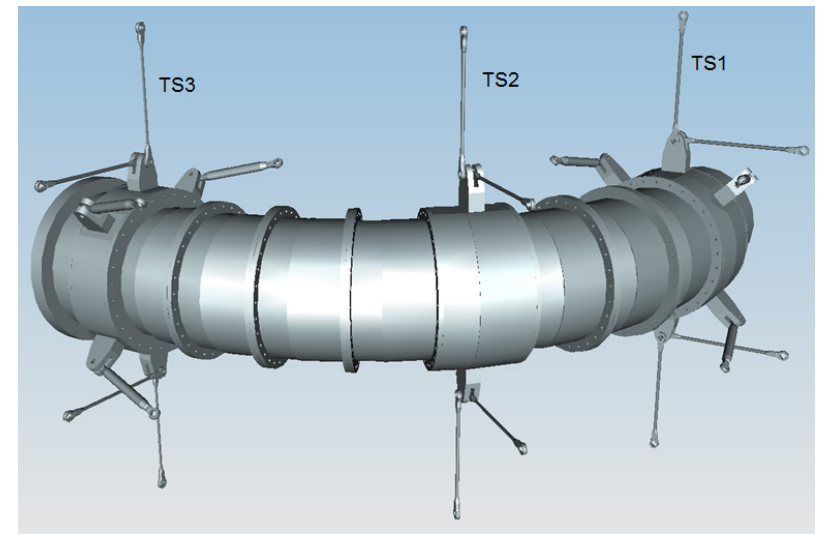

Fig. 8. TSu cold mass with the supports.

\section{B. Correction coils}

Another method that was investigated was the use of correction coils. A dipole winding could be placed on top of the first straight section (Figure 9). The interesting thing about the correction coils for solenoid systems is that for a vertical beam displacement, one has to apply a vertical field, unlike beam in accelerator where a vertical field displaces the beam horizontally. A $0.1 \mathrm{~T}-\mathrm{m}$ integrated field from the correction coil will result in around $40 \mathrm{~mm}$ beam displacement (Figure 10). This displacement, again, is independent of the particle momentum.

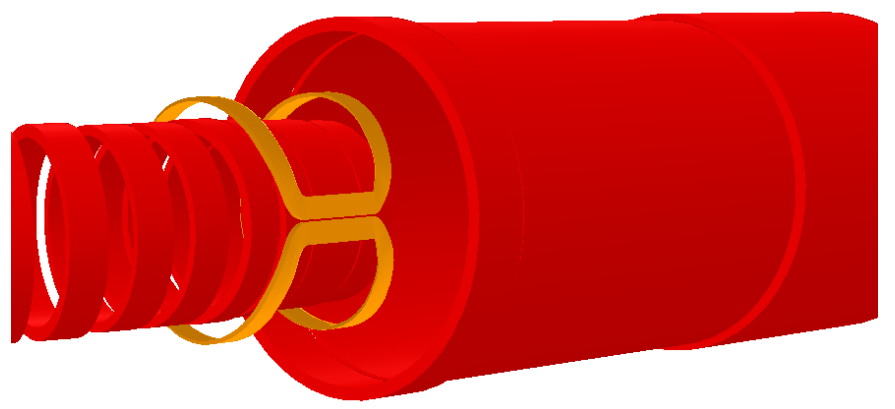

Fig. 9. A vertical correction coil model.

The disadvantage of a correction coil is the considerable complication in the design of the magnetic system. The needed integrated field is relatively high and its location (in the PS fringe field) would subject the coils to high forces. The mechanical support would be complicated. The quench 
protection will be also an issue. All result in a significant increment in the overall cost of the magnet.

\section{Trim power supplies}

Another option to tune the beam position is the use of trim power supplies (TPS). Those devices typically allow changes of a few percent of the main current. Figure 11 shows, schematically, the coils where a TPS is employed.

In order to allow $105 \mathrm{MeV}$ electrons to be displaced upwards, one must reduce the current on the coils. A reduction of $10 \%$ of the current results in these electrons moving around $20 \mathrm{~mm}$ up. An interesting fact is that this kind of modification is momentum-dependent; the LMCP technique does not apply in this particular case. $105 \mathrm{MeV}$ electrons were tracked instead. Table II summarizes the results of the horizontal and vertical displacements (with respect to the coils in nominal conditions) of electrons with different energies.

Displacement with respect to the nominal track
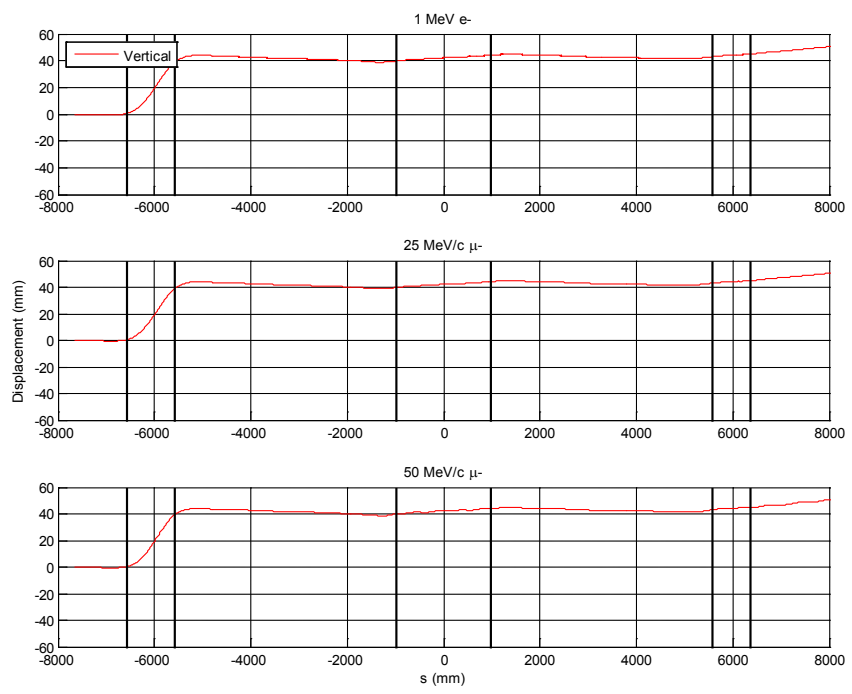

Fig. 10. Vertical beam displacement using a correction coil located in the first straight section.

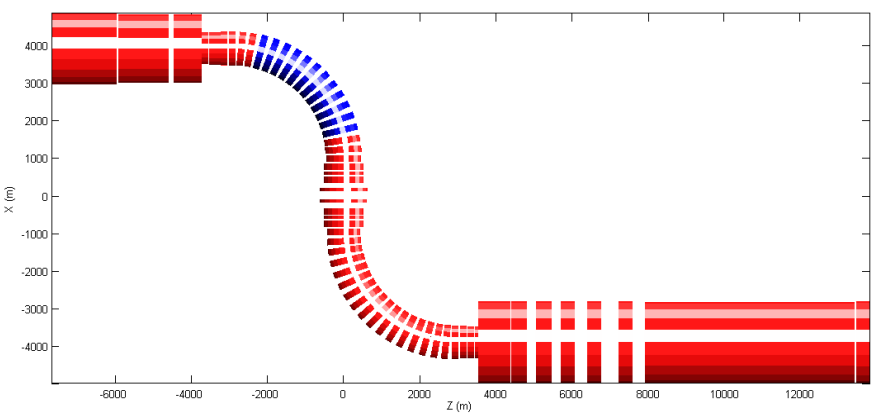

Fig. 11. Mu2e solenoid system. The coils in blue represent the ones powered with trim power supplies.

TABLE II

VERTICAL AND HORIZONTAL DISPLACEMENT USING TRIM POWER SUPPLIES. UNITS ARE mm.

\begin{tabular}{lcc}
\hline & $105 \mathrm{MeV}$ & $1 \mathrm{MeV}$ \\
\cline { 2 - 3 } Vertical & 19.0 & -1.4 \\
Horizontal & -1.4 & 3.1 \\
\hline \hline
\end{tabular}

\section{CONCLUSION}

Tracking studies have shown that the beam follows the magnetic center. A technique to determine the magnetic center of the Mu2e solenoid system was discussed. The technique is useful to predict displacements and indicate corrections to the magnetic design in a reliable and fast way. The technique was also used to estimate the mechanical tolerances of the support structures. The most restrictive tolerance has to do with the angles of the coils (yaw and pitch). The mechanical tolerances were fixed at $0.2^{\circ}$ in order to keep the RMS displacements lower than $3 \mathrm{~mm}$.

The magnetic center can be measured in the same way as presented here. The use of a beta source or even a cathode installed in the same location as the production target in PS is planned to be used [6]. The position could be measured with a detector in between TSu and TSd. In the event that corrections are needed, a controlled misalignment of TSu can provide the necessary vertical displacement. In that case a vertical centering force will push the magnet back to the center. The supports are design to withstand those additional forces.

During the commissioning of the experiment, a trim power supply (connected to the majority of the coils that compose the first curve) can be used on-the-fly for calibration purposes.

\section{REFERENCES}

[1] Mu2e Collaboration, "Mu2e Conceptual Design Report", arXiv:1211.7019, http://arxiv.org/abs/1211.7019

[2] V. V. Kashikhin et al., "Conceptual Design of the Mu2e Production Solenoid Cold Mass," Advances in Cryogenic Engineering, AIP Conf. Proc., 1434, 893-900 (2012).

[3] G. Ambrosio et al. - "Challenges and Design of the Transport Solenoid for the Mu2e experiment"- this conference;

[4] S. Feher et al. - "Reference Design of the Mu2e Detector Solenoid" this conference.

[5] J. Miller, R. Coleman Reference.

[6] M. Buehler et al. - "Mu2e Magnetic Measurements", this conference (3PoAJ-12) 